\title{
Intermediate hepatocellular carcinoma: the role of transarterial therapy
}

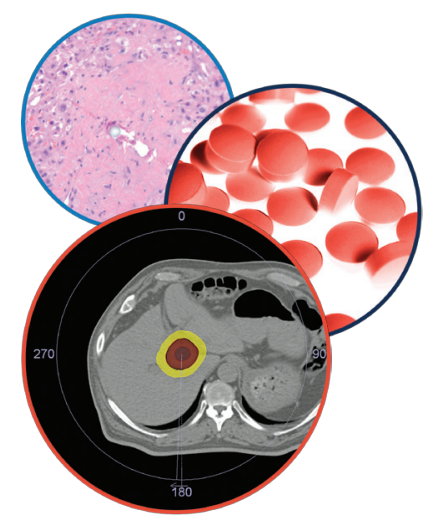

Hepatic Oncology

\author{
Fabrizio Chegai ${ }^{1,2}$, Antonio Orlacchio', Stefano Merolla', Serena Monti ${ }^{3}$ \\ \& Lorenzo Mannelli,2
}

\section{Practice points}

- Transarterial therapy is considered the gold standard for intermediate-stage hepatocellular carcinoma.

- It is important to know different modalities of transarterial therapy to perform a tailored approach for every patient.

- Multiple large series reported efficacy data with transarterial embolization, conventional transarterial chemoembolization, transarterial chemo-occlusion and radioembolization including downstaging to resection or transplantation in some patients.

- Careful, multidisciplinary patient selection is required to achieve the best outcomes.

- To minimize the risk of uncommon but potentially major adverse events, transarterial therapy should be administered by an experienced interventional radiologist.

According to Barcelona Clinic Liver Cancer, the recommended first-line treatment for patients with intermediate stage of hepatocellular carcinoma $(\mathrm{HCC})$ is transarterial chemoembolization. Patients with intermediate stage of HCC represent $20 \%$ with a 2-year survival of approximately $50 \%$. Nowadays, transarterial therapies have proved precious in the treatment of hepatic malignancies. During the last years, there were important developments in practiced transarterial therapies and their efficacy is still controversial. The purpose of this review is to discuss in further details these transarterial therapies that have been used to treat cases of HCC.

\section{Background}

Nowadays, the Barcelona Clinic Liver Cancer (BCLC) staging system is widely accepted in clinical practice and is used for many clinical trials of new drugs to treat hepatocellular carcinoma (HCC) [1].

Prognosis of patients affected by HCC depends on tumor stage at the time of diagnosis [2].

Only $30-40 \%$ of patients are being diagnosed at early stages when potentially curative treatments could be applied. Intermediate-stage HCC or stage B according to BCLC consists of multinodular tumors in patients with Child-Pugh A or B cirrhosis and good performance status [3].

Patients classified as BCLC-B, or intermediate-stage HCC, represent the $20 \%$ of HCC patients with a 2-year survival of approximately $50 \%$ [1].

\section{KEYWORDS}

- chemoembolization

- DEB TACE • embolization

- hepatocellular carcinoma

- interventional treatment

- radioembolization

- transarterial chemo-

occlusion

- transarterial therapy

\section{Future Medicine ${ }_{\text {part of }}$}


According to BCLC, the recommended firstline treatment for these patients is transarterial chemoembolization (TACE) [4], using in-oil anticancer emulsion (Lipiodol ${ }^{\circledR}$-TACE), which also is the most common transarterial techniques used in the HCC treatment.

However, important developments occurred in the most recent years in practiced transarterial therapies. These constitute TACE with or without drug-eluting beads (DEB), bland embolization, transarterial chemotherapy, transarterial chemo-occlusion (TACO) and transarterial radioembolization [5-7]. Even if the mechanism of achieving local tumor destruction is different in these types of transarterial therapy, hepatic artery embolotherapy with chemotherapy or without chemotherapy represents the standard of care for unresectable HCC [8].

Patient treatment should be chosen after evaluation of a multidisciplinary team composed of radiologist, oncologist, hepatologists, surgeons and interventional radiologists [9].

The purpose of this review is to discuss in further details these transarterial therapies that have been used to treat cases of HCC.

Rationale of transarterial therapies

The normal liver receives a dual blood supply from the hepatic artery (25\%) and the portal vein $(75 \%)$. During carcinogenesis, the normal liver blood supply changes. HCC becomes increasingly 'arterialized' and as a result of neoangiogenesis, the hepatic artery becomes its sole supplier [10]. Once a tumor nodule reaches a diameter of $2 \mathrm{~cm}$ or more, most of the blood supply derives from the hepatic artery. This unique property of HCC provides the rationale for widely used transarterial strategy to administer embolizing agents to achieve local tumor treatment.

\section{Transarterial embolization (bland} embolization)

The concept of embolization of liver neoplasms by blocking their arterial blood supply was introduced in the 1950s [11] and Doyon et al. described the first cases of bland embolization in the treatment of unresectable liver tumors [12]. Changes of tumor vascularization during carcinogenesis allow performing transarterial embolization (TAE) by delivering selective therapy to cancerous cells avoiding ischemic necrosis of the normal liver tissue [13]. The goal of TAE is to cause cell necrosis by blocking tumor vascularity without the administration of systemic chemotherapy. Some commonly used embolizing agents include gelatin sponge particles, polyvinyl alcohol particles (PVA) and polyacrylamide microspheres $[8,14,15]$. Gelatin sponge particles are a suboptimal embolizing agent, due to the large size of the particles $(1 \mathrm{~mm})$ and the temporary occlusion of the tumor-feeding arteries that only lasts for 2 weeks [16]. Gelfoam is an absorbable gelatin powder from absorbable gelatin sponge.

PVA particles provide more permanent arterial occlusion and can potentially provide more distal arterial obstruction as their size can be as small as 45-150 $\mu \mathrm{m}$ [17].

In most of the published studies on TAE for $\mathrm{HCC}$, gelatin sponge is commonly used embolic agent and it was also employed in a group of patients treated with TAE in the randomized clinical trial reported by Llovet et al. [18]. Even though neither PVA nor gelfoam has superior survival benefits over the other, Brown et al. showed that the number of TAE sessions was significantly greater for the gelfoam powder group (mean: 2.2) versus the PVA group (mean: 1.6; $\mathrm{p}=0.01)$ [19].

Few reports have assessed the outcomes of TAE for HCC [20-22]. Brown et al. [20] using regular PVA in $46 \mathrm{HCC}$ patients reported that after repeated TAE was possible to obtain 1- and 2 -year survival of 50 and $33 \%$, respectively. In 2008, Osuga et al. [23] using superabsorbent polymer microspheres (SAP TAE) treated 59 HCC patients for a total of 121 repeated SAP TAE. Authors demonstrated the safety of repeated procedures describing few cases of postembolization syndrome (PES) with no major complications. The median survival period was 30 months. Overall survival (OS) rates at 1 - and 2-year intervals were 100 and $83 \%$, respectively. Rand et al. [24] reported the result of a total of 106 TAEs using 100 - to 700- $\mu \mathrm{m}$ embospheres combined with proximal permanent occlusion with cyanoacrylate in 46 patients with unresectable HCC. The 180-, 360-, 520and 700-day cumulative survival rates were 81 , 71,71 and $47 \%$, respectively, with a median survival of 666 days. Covey et al. using 40- to $120-\mu \mathrm{m}$ trisacryl gelatin microspheres in TAE for postoperatively recurrent HCC reported a 1-, 2- and 5-year survival rates of 86, 74 and $47 \%$, respectively, with a median survival time of 46 months.

Maluccio et al. [25] performed selective embolization of arterial vessels feeding the 
hepatic tumors with spherical embolic particles $(40-120 \mu \mathrm{m})$ or small $(50 \mu \mathrm{m})$ PVA intended to block the terminal vessels in 322 patients. Authors reported survival rates of $66 \%$ at 1 year, $46 \%$ at 2 years and $33 \%$ at 3 years, while the median survival for the entire sample was 21 months.

The most common toxic effects of TAE are usually associated with the PES consisting of fever, nausea, abdominal pain and vomiting [26]. TAE can be safely repeated to achieve maximal tumor response and limit disease progression.

It is well known that arterial occlusion, both proximal and distal, can lead to development of collateral vessels [27] and this event could limit the ability to repeat embolization via conventional hepatic vessels. Erinjeri et al. [15] performed repeated TAE in 43 patients, using 'permanent' embolic agents (PVA or microspheres), and demonstrated that patency of the hepatic arterial tree is preserved in $>80 \%$ of patients even after an average of $6 \pm 2$ procedures during a mean treatment period of $47 \pm 24$ months.

The studies referred to by Brown, Covey, Maluccio and Erinjeri are all from the same hospital/group.

It is not clear which embolic agent is the most effective in TAE, and available data showed great variability in terms of safety, repeatability, tumor response and survival. This variability could be explained by the heterogeneity of patients' populations, etiology of underlying cirrhosis, embolization technique, choice of particle size and outcome assessment method.

\section{Transarterial chemoembolization}

\section{- Conventional TACE}

It is well established that HCC is a relatively chemoresistant tumor and systemic chemotherapy has poor effects on it [28]. The first report on the use of anticancer drugs followed by gelfoam was reported by Yamada $e t$ al. in the late 1970s [29]. The added value of chemotherapeutic agents during transarterial HCC embolization was discussed [30]. Combination of embolization and chemotherapeutic agents is used to prevent washout of the drug at the site of the tumor; this allows for a better absorption of chemotherapeutic agents and combined the effects of chemotherapy and ischemic necrosis [31]. However, there are not standard protocols defined for TACE and several anticancer drugs are used, including doxorubicin [18], cisplatin [32] and epirubicin [33]. Typically, the chemotherapy agent is emulsified in Lipiodol.

Lipiodol is an oily contrast agent used to increase intratumoral retention of the cytotoxic agent [34,35]. Lipiodol is retained into the tumor and not into the liver parenchyma because of the absence of Kupffer in HCCs and hemodynamic differences between HCCs and liver parenchyma [36,37].

There are no standard protocols of TACE universally adopted and there are variations in the procedure done and the drugs used. Single or combination of chemotherapeutic agents can be used, depending on operator preference and tumor type. The dose of chemotherapeutic agent also varies among centers. In published trials, the median dose of doxorubicin, cisplatin and epirubicin administered per session was 50, 92 and $50 \mathrm{mg}$, respectively [8].

The main studies on the use of TACE in selected patients with well-preserved liver function were carried out in 2002 by Llovet et al. [18] and Lo et al. [32].

The trial conducted by Llovet et al. was stopped when the ninth sequential inspection showed that chemoembolization had survival benefits compared with conservative treatment. However, survival probabilities at 1 and 2 years were 75 and $50 \%$ for TAE and 82 and $63 \%$ for conventional TACE (cTACE) without statistically differences.

Lo et al. showed a statistically significant survival advantage with the use of TACE versus TAE or symptomatic treatment. In 2003, Llovet and Bruix publishing a systematic review of randomized trials for unresectable HCC reported a significant benefit of chemoembolization with cisplatin or doxorubicin but none with embolization alone [38].

According to the authors of both studies, TACE could be considered the best techniques to improve survival of selected patients with unresectable HCC.

Takayasu et al. [39] treated $8510 \mathrm{HCC}$ patients with TACE (Lipiodol, chemotherapy and gelatin sponge particles) and reported an overall median survival of 34 months, with survival rates of $82 \%$ at 1 year, $47 \%$ at 3 years, $26 \%$ at 5 years and $16 \%$ at 7 years. In this study, liver function, TNM stage and AFP values were independent risk factors for patient survival.

In 2009, Zhou et al. [40] evaluated the effect of preoperative TACE for resectable large HCC in 108 patients, randomly assigned to 
preoperative TACE treatment or not (control group). Zhou et al. reported no significant difference between the two groups in operative blood loss, surgical morbidity and hospital mortality. The preoperative TACE group had a lower resection rate and longer operative time, and at a median follow-up of 57 months, 41 (78.8\%) of 52 patients in the preoperative TACE group and $51(91.1 \%)$ of 56 patients in the control group had recurrent disease. The 1-, 3- and 5-year disease-free survival rates were 48.9, 25.5 and $12.8 \%$, respectively, for the preoperative TACE group and 39.2, 21.4 and $8.9 \%$, respectively, for the control group. Authors concluded that preoperative TACE did not improve surgical outcome.

It is accepted that different characteristics of patient-, tumor- and treatment-types could be associated with better survival after TACE, but results from individual studies are sometimes conflicting [4,41]. Recently Biolato et al. [42] analyzed the prognostic role of baseline clinical, biochemical and radiological characteristics of 270 patients with HCC treated with TACE. Authors showed how nontumor segmental portal vein thrombosis (PVT), serum sodium, diameter of largest nodule, number of nodules, AFP and ALP were independent prognostic factors for OS on multivariate analysis. Biolato et al. concluded that serum sodium should be included of alongside the already known prognostic factors and it may allow a better prognostic definition of patients with HCC as candidates for cTACE.

According to a systematic review, TACE treatment-related mortality was $2.4 \%$ (range: $0-9.5 \%$ ) in 37 trials involving 2858 patients [8]. As described for TAE, PES is the most common side effect after Lipiodol TACE, but a systemic toxicity induced by doxorubicin is reported in 60\% of patients [8].

\section{- TACE with drug-eluting microspheres}

The goal of TACE is that a maximum and sustained concentration of the chemotherapeutic agent must be obtained into the tumor limiting to a minimum the systemic exposure [43].

The concept of DEB is similar to conventional chemoembolization but the introduction of the latter has provided an attractive alternative to TACE. DEB allow to maximize the drug's effectiveness in terms of response with significantly reducing its systemic toxicity [44]. Hong et al. reported a significant reduction of chemotherapy agent peak plasma concentrations when using
DEB rather than Lipiodol. This suggests that a larger amount of the chemotherapy agent is being sequestered by the tumor instead than distributed in the systemic circulation [45].

There are different types of drug-eluting microspheres. Doxorubicin-capable bead or DC beads can be loaded with doxorubicin on hydrated beads by immersing them in a drug solution for 1-120 min [46,47]. Initial experiments with doxorubicin-loaded QuadraSphere ${ }^{\circledR}$ microspheres have shown a safe pharmacokinetic profile and effective tumor killing in an animal model of liver cancer [48].

The first clinical studies with TACE using DC beads (DEBTACE) in the treatment of HCC showed a high index of tumor necrosis, a low incidence of toxicities and overall response rates varying between 50 and 75\% [49-51]. Varela et al. [50] used DC beads in $27 \mathrm{HCC}$ patients and reported a response rate of $66.6 \%$.

In the USA, a Phase II study on the use of DC beads for HCC was published in 2009. Reyes et al. enrolled 20 HCC patients who underwent 34 treatment sessions. RECIST and EASL response rates at 1 month were 10 and $60 \%$, respectively. The remainder of patients had stable disease. OS rates at 1 and 2 years were 65 and 55\%, respectively; the median OS was 26 months. The authors concluded that chemoembolization with DC beads is safe and effective in achieving local tumor control inpatients with unresectable HCC [51,52].

In 2010, Lammer et al. conducted a multicenter study to evaluate the safety and efficacy of DC Bead with doxorubicin (DEB doxorubicin $[\mathrm{DEBDOX}]$ ) in the treatment of HCC in comparison with cTACE. Authors claimed that a trend toward higher response rates was observed for DC bead over CTACE [53].

The administered beads may vary according to the disease volume and distribution. The majority of previous studies used beads with size range between 100-300 and 300-500 $\mu \mathrm{m}$ [54].

Recently, Prajapati et al. [55] investigated the OS, efficacy and safety of small versus large DC beads TACE in patients with HCC. The authors evaluated 94 consecutive patients divided in two groups: group A (59 patients) using 100-300 $\mu \mathrm{m}$ beads, and group B (35 patients) using mixed 300-500 and 500-700 $\mu \mathrm{m}$ beads. The overall median survival in groups A and B were 15.1 and 11.1 months, respectively. Both groups were similar in demographics, tumor burden and differential staging $(\mathrm{p}>0.5)$. The particle size, 
Child-Pugh class and serum AFP level were significant prognostic indicators of survival on multivariate analysis. Thus, the authors concluded that TACE with 100-300- $\mu \mathrm{m}$-sized DEB is associated with significantly higher survival rate and lower complications than TACE with 300-500 and 500-700- $\mu \mathrm{m}$-sized DEB.

Systemic side effects of doxorubicin (alopecia, skin discoloration, mucositis and bone marrow suppression) are significantly lower for DC bead (11.8\%) over cTACE (25.9\%) [53].

\section{Transarterial chemo-occlusion}

Although TACE could be considered the best treatment option for intermediate-advanced HCC, the long-term survival of patients treated with TACE is not yet fully satisfactory [56].

Different conditions could explain this evidence, including differences in patient's selection or in tumor characteristics. Moreover, interrupting blood flow to the tumor, TACE surely induces necrosis at the site of disease, but may create conditions that facilitate VEGFmediated angiogenesis [57], which could be a further mechanism causing recurrence after TACE [58-60].

Thus, transient blood flow obstruction could be desirable allowing for repeated chemoembolization [61], especially because extended ischemia may precipitate new tumor vessel induction via VEGF stimulation [62].

Forsberg in 1978 reported the use of an amilomere (starch) microspheres with an average sphere diameter of $50 \mathrm{~lm}$ (range: 20-200 lm) in a sterile saline suspension. These particles have an half-life of 35-50 min due to degradation by serum a-amylase [63].

Therefore, to avoid an overexpression of VEGF, transient occlusion of tumor feeding artery using degradable starch microspheres (DSM)s was proposed [64] due to the possibility to obtain a short-term nonpermanent vascular occlusion. In fact, the main differences between cTACE and TACO can be found in the fact that DSMs are not retained into the tumor (as Lipiodol) and they have an half-life shorter than gelfoam.

Recently, Pieper et al. [6] evaluated the embolic properties, time to reperfusion and histologic changes in temporary embolization of liver tissue with DSMs in a swine model. Temporary embolization of the hepatic artery using DSMs is feasible with complete reperfusion after $30 \mathrm{~min}$ in pigs. Even after complete arterial blood flow stasis, no extensive tissue damage to the embolized liver parenchyma was observed at histologic examinations in this animal study.

To date, findings have primarily shown DSMs to be an effective short-term embolization method for TACE [65]. However, until now the clinical usefulness of DSMs in association with chemotherapeutic agents has been investigated only in few studies in patients with unresectable HCC, with highly contradictory results [66-70].

Furuse et al. [66], in 2003, reported encouraging results in a pilot study including 17 patients with multifocal HCC treated with repeated DSMs-TACO performed with a mixture of DSMs and epirubicin, obtaining a complete response in $11.8 \%$ and a partial response in $41.2 \%$. This group also reported less adverse effects of DSMs-TACO on liver function compared with Lipiodol-TACE.

Kirchhoff et al. [67], compared DSMs-TACO versus transarterial chemoperfusion, using in both cases a doxorubicin and cisplatin mixture, within a randomized study of 70 patients with advanced HCC, showing no differences in tumor response rates. Notably, this group found no evidence of complete response in both arms, and partial response of 26 and $9 \%$, respectively [68].

More recently, Yamasaki et al. [69] performed a prospective randomized trial to compare the efficacy of a single arterial infusion (TAI) chemotherapy using combined Lipiodol and DSMs versus both Lipiodol-TAI or DSMs-TAI, in 45 cirrhotic (both CPT stages A and B) patients with mono- or multi-lobar HCC (tumor size range: $27-33 \mathrm{~mm}$ ). The study showed a trend toward a greater tumor response rate in the combined Lipiodol/DSMs-TAI group (complete response $=40 \%)$ compared with the two monotherapy groups (Lipiodol-TAI: complete response $=26.7 \%$ and DSMs-TAI: complete response $=26.7 \%$ ). The progression-free survival was higher in the combined Lipiodol/DSMs-TAI group (377 days) compared with the DSMs-TAI (287 days; $\mathrm{p}=0.02$ ) or Lipiodol-TAI (177 days; $\mathrm{p}=0.03)$ groups.

In 2014, Niessen et al. [70] compared outcomes of DSMTACO and cTACE in patients with unresectable intermediate-stage HCC. For the DSM TACO group, the objective response rate (i.e., complete or partial response) was $44.1 \%$, and the rate of stable disease was $38.2 \%$. The respective rates for the cTACE group were 48.6 and $31.4 \%$. Complications did not significantly 
differ between groups. Authors concluded that results of DSM chemoembolization were similar to those of conventional chemoembolization.

Taken together, these data suggest that the inclusion of DSMs in the TACE procedure may offer some advantage in the treatment of patients with unresectable HCC nodules smaller than $5 \mathrm{~cm}$ in diameter.

It may be possible to speculate that the short-term arterial occlusion obtained using DSMs-TACO, followed by a rapid tissue reperfusion (DSMs half-life is only $35-50 \mathrm{~min}$ ), could be able to limit hypoxia and acidosis and the consequent VEGF overproduction. The latter is thought to be a trigger for rebound neovascularization, tumor regrowth and cancer recurrence after radical or palliative HCC treatment.

As with TAE and cTACE, the most common side effect of TACO is PES.

\section{Radioembolization}

Historically, external beam irradiation played a limited role in the treatment of HCC because of the radiosensitive nature of normal hepatic tissue [71]. An estimated dose $\geq 70 \mathrm{~Gy}$ is required to cause necrosis of solid tumor, which is greater than the liver tolerance dose of 35 Gy [72].

Yttrium-90 radioembolization (Y90RE), defined as the injection of micron-sized embolic particles loaded with a radioisotope by using percutaneous transarterial techniques [73], is a novel transarterial approach to radiation therapy for liver cancer. In few retrospective studies, when compared with cTACE, Y90RE would seem to offer an advantage in survival, time-to-progression (TTP), toxicity [74] and efficacious tumor control also in patients with PVT $[75,76]$.

Two radioembolic agents are commercially available: TheraSpheres ${ }^{\circledR}$ (Nordion, Ottawa, Canada) and SIR Spheres ${ }^{\circledR}$ (Sirtex Medical Inc., MA, USA).

A consensus panel, consisting of professionals from interventional radiology, nuclear medicine, radiation oncology, medical and surgical fields has to determine the criteria for selecting patients suitable for radioembolization (RE) [78]. Contraindications include total serum bilirubin greater than $2 \mathrm{mg} / \mathrm{dl}$, excessive tumor size with poor hepatic function and a compromised portal vein. However, the lack of prospective Phase II investigations has limited a precise identification of a specific population of patients with HCC who may benefit from Y90RE as a first-line treatment [76]. From September 2003 to February
2005, Sangro et al. [79] treated 24 consecutive patients with HCC with RE to investigate antitumor effect and safety in the setting of liver cirrhosis. The authors discussed the importance of patients selection and calculation of Y90 activity dose to be administered.

A different and more disquieting syndrome may be observed when whole-body irradiation together with ablative doses of chemotherapy is used before bone marrow transplantation. In this so-called combined modality-induced liver disease (CMILD), patients develop jaundice, ascites and fluid retention and their blood tests mainly show increased bilirubin levels.

After external beam irradiation, there is high risk of developing radiation-induced liver disease (RILD), a clinical syndrome characterized by an anicteric hepatomegaly, ascites and elevated liver enzymes, especially alkaline phosphatase [80].

Similar to RILD, RE-induced liver disease (REILD) has been defined as jaundice and ascites appearing 1-2 months after RE in the absence of tumor progression or bile duct occlusion. REILD is an uncommon, but clinically relevant, complication of RE that tends to occur when a large volume of liver tissue usually harboring an unfavorable milieu as a result of cirrhosis, prior or subsequent chemotherapy is exposed to radiation-delivered radioactive microspheres. It may also appear when partial liver volumes are treated in patients with cirrhosis with a reduced liver functional reserve [81].

Salem et al. [73] treated 43 HCC patients with $90 \mathrm{Y}$ microspheres over a 4-year period. Patients were stratified into three groups according to method of treatment and risk stratification (group 0 : segmental; group 1: lobar low-risk; group 2: lobar high-risk) and the Okuda and Child-Pugh scoring systems. The authors reported no statistical difference among the three risk groups with respect to tumor response. Twenty patients (47\%) had an objective tumor response based on percent reduction in tumor size and 34 patients $(79 \%)$ had a tumor response when percent reduction and/or tumor necrosis were used as a composite measure of tumor response. Survival times from date of diagnosis were different among the risk groups $(\mathrm{p}<0.0001)$. Median survival times were 46.5, 16.9 and 11.1 months for groups 0,1 and 2, respectively. The authors reported no life-threatening complications related to treatment.

Recently, Mazzaferro et al. [76] reported their results on 52 patients with intermediate and advanced HCC treated with Y90RE. The authors 
analyzed as the primary end point the efficacy of Y90RE on TTP and as secondary end points tumor response, safety and OS.

The median follow-up was 36 months. Five complete responses occurred $(9.6 \%)$, and the 2 year-progression rate was $62 \%$. Tumor response significantly correlated with absorbed dose in target lesions and a threshold of 500-Gy-predicted response. The median TTP was 11 months with no significant difference between PVT and no PVT (7 vs 13 months). The median OS was 15 months with a nonsignificant trend in favor of non-PVT versus PVT patients (18 vs 13 months).

For the authors, tumor response was the sole variable affecting TTP and the second affecting survival (after the Child-Pugh class).

El Fouly et al. [82] collected data of 86 HCC patients in two university hospitals where cTACE with doxorubicin or TARE Y-90 using glass microspheres was performed. Despite a higher tumor burden in the TARE Y-90 group, the median OS and the median TTP were not statistically different. The number of treatment sessions, the average rate of treatment sessions per patient, total hospitalization time and rate of adverse events were significantly higher in the TACE cohort. For the authors, TARE Y-90 was better tolerated and associated with less hospitalization and treatment sessions. Moreover, both treatments resulted in similar survival probabilities despite more advanced disease in the TARE Y-90 group.

In 2013, Kim et al. [83] performed a multicentric prospective study to reveal the efficacy and safety of TARE Y-90 in 42 HCC patients. Median follow-up was 29 months. At 3 months, the complete response, partial response and stable disease were seen in $4(10.0 \%), 19(47.5 \%)$ and $15(37.5 \%)$ patients, respectively. The response rate was $57.5 \%(23 / 40)$, and disease control rate was $95 \%(38 / 40)$ at 3 months. The response rate at 6 months was $63.9 \%(23 / 36)$, and disease control rate was $83.3 \%$ (30/36). The median time to progression was 18 months.

Previous results had been shown that there are different predictors of survival in patients treated with transarterial therapies. TTP and survival varied by tumor stage and liver function [74]. Several studies have investigated the effect of inflammation on carcinogenesis because the cytokines and mediators released by inflammatory cells can promote angiogenesis and tumor cell metastasis [84,85]. The neutrophil-lymphocyte ratio (NLR) is a simple biomarker of inflammation, and an elevated NLR has been linked to several malignancies [86,87].
In 2015, Sukato et al. [88] reported results about the prognostic value of NLR in patients with HCC treated with Y90RE. In their work, patients with a normal NLR were found to have longer survival than individuals with a high NLR in intermediate/advanced-disease and advanced-disease cohorts. Elevated NLR, high $\alpha$-fetoprotein level and low albumin level were independent predictors of worse survival. For the authors, patients with BCLC-stage $\mathrm{C}$ disease with elevated NLR may not derive benefit from RE.

\section{Conclusion}

Transarterial therapies including bland embolization, chemoembolization, chemo-occlusion and $\mathrm{RE}$ are effective in the treatment of intermediate HCC. Patient selection for one treatment option is essential to optimize the outcome. Direct comparison of these and other treatment options in HCC is difficult to obtain. More studies are needed to determine the effects of these treatments alone and in combination with other treatments.

Transarterial therapies demonstrated minimal toxicity profiles and highly effective tumor responses, and spear the normal hepatic parenchyma from treatment.

\section{Future perspective}

The number of patients with primary liver tumor are continuously increasing and it is desirable a development of new technologies requiring a steady collaboration of difference specialists with multidisciplinary approach for a right management. Therapies should be based on scientifically supported guidelines and at the same time should be designed to suit the individual patient. The encouraging evidence supporting the potential role of all different transarterial therapies in the management of intermediate HCC also highlights the many unanswered questions and challenges, underscoring the need for further prospective studies.

Financial \& competing interests disclosure

This manuscript was supported by Memorial Sloan Kettering Cancer Center under the institutional NIH grant: P3O CA008748. The authors have no other relevant affiliations or financial involvement with any organization or entity with a financial interest in or financial conflict with the subject matter or materials discussed in the manuscript apart from those disclosed.

No writing assistance was utilized in the production of this manuscript. 


\section{References}

Papers of special note have been highlighted as: - of interest; $\bullet \bullet$ of considerable interest

1 European Association for the Study of the Liver, European Organisation for Research and Treatment of Cancer. EASL-EORTC clinical practice guidelines: management of hepatocellular carcinoma. J. Hepatol. 56(4), 908-943 (2012).

-• Reports the standardized guidelines for the hepatocellular carcinoma (HCC) treatment.

2 Kim WR, Gores GJ, Benson JT, Therneau TM, Melton LJ. Mortality and hospital utilization for hepatocellular carcinoma in the United States. Gastroenterology 129(2), 486-493 (2005).

3 Tsochatzis E, Meyer T, O’Beirne J, Burroughs AK. Transarterial chemoembolisation is not superior to embolisation alone: the recent European Association for the Study of the Liver (EASL) - European Organisation for Research and Treatment of Cancer (EORTC) guidelines. Eur. J. Cancer 49(6), 1509-1510 (2013).

4 Raoul J-L, Sangro B, Forner A et al. Evolving strategies for the management of intermediate-stage hepatocellular carcinoma: available evidence and expert opinion on the use of transarterial chemoembolization. Cancer Treat. Rev. 37(3), 212-220 (2011).

5 Lewandowski RJ, Geschwind J-F, Liapi E, Salem R. Transcatheter intraarterial therapies: rationale and overview. Radiology 259(3), 641-657 (2011).

6 Pieper CC, Meyer C, Vollmar B, Hauenstein K, Schild HH, Wilhelm KE. Temporary arterial embolization of liver parenchyma with degradable starch microspheres (EmboCept ${ }^{\circledR} S$ ) in a swine model. Cardiovasc. Intervent. Radiol. 38(2), 435-441 (2015).

- Evaluates the real temporary effect of degradable starch microspheres.

7 Deipolyi AR, Oklu R, Al-Ansari S, Zhu AX, Goyal L, Ganguli S. Safety and efficacy of 70-150 $\mu \mathrm{m}$ and $100-300 \mu \mathrm{m}$ drug-eluting bead transarterial chemoembolization for hepatocellular carcinoma. J. Vasc. Interv. Radiol. 26(4), 516-522 (2015).

8 Marelli L, Stigliano R, Triantos C et al. Transarterial therapy for hepatocellular carcinoma: which technique is more effective? A systematic review of cohort and randomized studies. Cardiovasc. Intervent. Radiol. 30 (1), 6-25 (2007).

9 Gish RG, Lencioni R, Di Bisceglie AM, Raoul JL, Mazzaferro V. Role of the multidisciplinary team in the diagnosis and treatment of hepatocellular carcinoma. Expert
Rev. Gastroenterol. Hepatol. 6(2), 173-185 (2012).

- Reports the important role of a multidisciplinary approach for a tailored treatment for each patient.

10 Markowitz J. The hepatic artery. Surg. Gynecol. Obstet. 95(5), 644-646 (1952).

11 Bierman HR, Byron RL, Kelley KH, Grady A. Studies on the blood supply of tumors in man. III. Vascular patterns of the liver by hepatic arteriography in vivo. J. Natl Cancer Inst. 12(1), 107-131 (1951).

12 Doyon D, Mouzon A, Jourde AM, Regensberg C, Frileux C. Hepatic, arterial embolization in patients with malignant liver tumours (author's transl.). Ann. Radiol. (Paris). 17(6), 593-603 (1974).

13 Gyves JW, Ziessman HA, Ensminger WD et al. Definition of hepatic tumor microcirculation by single photon emission computerized tomography (SPECT). J. Nucl. Med. 25(9), 972-967 (1984).

14 Bonomo G, Pedicini V, Monfardini L et al. Bland embolization in patients with unresectable hepatocellular carcinoma using precise, tightly size-calibrated, antiinflammatory microparticles: first clinical experience and one-year follow-up. Cardiovasc. Intervent. Radiol. 33(3), 552-559 (2010).

- Describes accurate tips and tricks for bland embolization.

15 Erinjeri JP, Salhab HM, Covey AM, Getrajdman GI, Brown KT. Arterial patency after repeated hepatic artery bland particle embolization. J. Vasc. Interv. Radiol. 21(4), 522-526 (2010).

16 Tsochatzis E, Meyer T, Marelli L, Burroughs AK. Which transarterial therapy is best for hepatocellular carcinoma? The evidence to date. J. Hepatol. 53(3), 588 (2010).

17 Coldwell DM, Stokes KR, Yakes WF. Embolotherapy: agents, clinical applications, and techniques. Radiographics 14(3), 623-643 (1994).

18 Llovet JM, Real MI, Montaña X et al. Arterial embolisation or chemoembolisation versus symptomatic treatment in patients with unresectable hepatocellular carcinoma: a randomised controlled trial. Lancet 359(9319), 1734-1739 (2002).

- Represents the first randomized trial on HCC transarterial chemoembolization.

19 Brown DB, Pilgram TK, Darcy MD et al. Hepatic arterial chemoembolization for hepatocellular carcinoma: comparison of survival rates with different embolic agents.
J. Vasc. Interv. Radiol. 16(12), 1661-1666 (2005).

-• Reports accurately on the differences between different embolic agents.

20 Brown KT, Nevins AB, Getrajdman GI et al. Particle embolization for hepatocellular carcinoma. J. Vasc. Interv. Radiol. 9(5), 822-828 (1998).

- Reports accurately on the important role of different embolic agents for the HCC treatment.

21 Maluccio M, Covey AM, Gandhi R et al. Comparison of survival rates after bland arterial embolization and ablation versus surgical resection for treating solitary hepatocellular carcinoma up to $7 \mathrm{~cm}$. J. Vasc. Interv. Radiol. 16(7), 955-961 (2005).

22 Covey AM, Maluccio MA, Schubert J et al. Particle embolization of recurrent hepatocellular carcinoma after hepatectomy. Cancer 106(10), 2181-2189 (2006).

23 Osuga K, Hori S, Hiraishi K et al. Bland embolization of hepatocellular carcinoma using superabsorbent polymer microspheres. Cardiovasc. Intervent. Radiol. 31(6), 1108-1116 (2008).

24 Rand T, Loewe C, Schoder M et al. Arterial embolization of unresectable hepatocellular carcinoma with use of microspheres, lipiodol, and cyanoacrylate. Cardiovasc. Intervent. Radiol. 28(3), 313-318 (2005).

25 Maluccio MA, Covey AM, Porat LB et al. Transcatheter arterial embolization with only particles for the treatment of unresectable hepatocellular carcinoma. J. Vasc. Interv. Radiol. 19(6), 862-869 (2008).

26 Oliveri RS, Wetterslev J, Gluud C. Transarterial (chemo)embolisation for unresectable hepatocellular carcinoma. Cochrane Database Syst. Rev. 3, CD004787 (2011).

27 Miyayama S, Matsui O, Taki K et al. Extrahepatic blood supply to hepatocellular carcinoma: angiographic demonstration and transcatheter arterial chemoembolization. Cardiovasc. Intervent. Radiol. 29(1), 39-48 (2006).

28 Kalayci C, Johnson PJ, Raby N, Metivier EM, Williams R. Intraarterial adriamycin and Lipiodol for inoperable hepatocellular carcinoma: a comparison with intravenous adriamycin. J. Hepatol. 11(3), 349-353 (1990).

29 Yamada R, Sato M, Kawabata M, Nakatsuka H, Nakamura K, Takashima S. Hepatic artery embolization in 120 patients with unresectable hepatoma. Radiology 148(2), 397-401 (1983). 
30 Chang JM, Tzeng WS, Pan HB, Yang CF, Lai $\mathrm{KH}$. Transcatheter arterial embolization with or without cisplatin treatment of hepatocellular carcinoma. A randomized controlled study. Cancer 74(9), 2449-2453 (1994).

31 Kruskal JB, Hlatky L, Hahnfeldt P, Teramoto K, Stokes KR, Clouse ME. In vivo and in vitro analysis of the effectiveness of doxorubicin combined with temporary arterial occlusion in liver tumors. J. Vasc. Interv. Radiol. 4(6), 741-747 (1993).

32 Lo CM, Ngan H, Tso WK et al. Randomized controlled trial of transarterial Lipiodol chemoembolization for unresectable hepatocellular carcinoma. Hepatology 35(5), 1164-1171 (2002).

33 Kawai S, Tani M, Okamura J et al. Prospective and randomized trial of Lipiodol-transcatheter arterial chemoembolization for treatment of hepatocellular carcinoma: a comparison of epirubicin and doxorubicin (second cooperative study). The Cooperative Study Group for Liver Cancer Treatment. Semin. Oncol. 24(2 Suppl. 6), S6-38-S6-45, (1997).

34 Bhattacharya S, Dhillon AP, Winslet MC et al. Human liver cancer cells and endothelial cells incorporate iodised oil. Br. J. Cancer 73(7), 877-881 (1996).

35 Terayama N, Matsui O, Gabata T et al. Accumulation of iodized oil within the non-neoplastic liver adjacent to hepatocellular carcinoma via the drainage routes of the tumor after transcatheter arterial embolization. Cardiovasc. Intervent. Radiol. 24(6), 383-387 (2001).

36 Kan Z, McCuskey PA, Wright KC, Wallace S. Role of Kupffer cells in iodized oil embolization. Invest. Radiol. 29(11), 990-903 (1994).

37 Kan Z, Wright K, Wallace S. Ethiodized oil emulsions in hepatic microcirculation: in vivo microscopy in animal models. Acad. Radiol. 4(4), 275-282 (1997).

38 Llovet JM, Bruix J. Systematic review of randomized trials for unresectable hepatocellular carcinoma: chemoembolization improves survival. Hepatology 37(2), 429-442 (2003).

-• Represents the first systematic review of randomized trials for unresectable HCC.

39 Takayasu K, Arii S, Ikai I et al. Prospective cohort study of transarterial chemoembolization for unresectable hepatocellular carcinoma in 8510 patients. Gastroenterology 131(2), 461-469 (2006).
40 Zhou WP, Lai ECH, Li AJ et al. A prospective, randomized, controlled trial of preoperative transarterial chemoembolization for resectable large hepatocellular carcinoma. Ann. Surg. 249(2), 195-202 (2009).

41 Grieco A, Marcoccia S, Miele L et al. Transarterial chemoembolization (TACE) for unresectable hepatocellular carcinoma in cirrhotics: functional hepatic reserve and survival. Hepatogastroenterology 50 (49), 207-212 (2003).

42 Biolato M, Miele L, Vero V et al. Hepatocellular carcinoma treated by conventional transarterial chemoembolization in field-practice: serum sodium predicts survival. World J. Gastroenterol. 20(25), 8158-8165 (2014).

43 Brown DB, Gould JE, Gervais DA et al. Transcatheter therapy for hepatic malignancy: standardization of terminology and reporting criteria. J. Vasc. Interv. Radiol. 20 (Suppl. 7), S425-S434 (2009).

- Reports standardization of terminology and reporting criteria for the HCC treatment.

44 Nicolini A, Martinetti L, Crespi S, Maggioni M, Sangiovanni A. Transarterial chemoembolization with epirubicin-eluting beads versus transarterial embolization before liver transplantation for hepatocellular carcinoma. J. Vasc. Interv. Radiol. 21(3), 327-332 (2010).

45 Hong K, Khwaja A, Liapi E, Torbenson MS, Georgiades CS, Geschwind JFH. New intra-arterial drug delivery system for the treatment of liver cancer: preclinical assessment in a rabbit model of liver cancer. Clin. Cancer Res. 12(8), 2563-2567 (2006).

46 Lewis AL, Gonzalez MV, Lloyd AW et al. DC bead: in vitro characterization of a drugdelivery device for transarterial chemoembolization. J. Vasc. Interv. Radiol. 17(2), 335-342 (2006).

47 Lencioni R, de Baere T, Burrel M et al. Transcatheter treatment of hepatocellular carcinoma with doxorubicin-loaded DC Bead (DEBDOX): technical recommendations. Cardiovasc. Intervent. Radiol. 35(5), 980-985 (2012).

48 Lee K-H, Liapi EA, Cornell C et al. Doxorubicin-loaded QuadraSphere ${ }^{\circledR}$ microspheres: plasma pharmacokinetics and intratumoral drug concentration in an animal model of liver cancer. Cardiovasc. Intervent. Radiol. 33(3), 576-582 (2010).

49 Malagari K, Alexopoulou E, Chatzimichail K et al. Transcatheter chemoembolization in the treatment of HCC in patients not eligible for curative treatments: midterm results of doxorubicin-loaded DC bead. Abdom. Imaging 33(5), 512-519 (2008).

50 Varela M, Real MI, Burrel M et al. Chemoembolization of hepatocellular carcinoma with drug eluting beads: efficacy and doxorubicin pharmacokinetics. J. Hepatol. 46(3), 474-481 (2007).

51 Poon RTP, Tso WK, Pang RWC et al. A Phase I/II trial of chemoembolization for hepatocellular carcinoma using a novel intra-arterial drug-eluting bead. Clin. Gastroenterol. Hepatol. 5(9), 1100-1108 (2007).

52 Reyes DK, Vossen JA, Kamel IR et al. Single-center Phase II trial of transarterial chemoembolization with drug-eluting beads for patients with unresectable hepatocellular carcinoma: initial experience in the United States. Cancer J. 15(6), 526-532 (2009).

53 Lammer J, Malagari K, Vogl Tet al. Prospective randomized study of doxorubicineluting-bead embolization in the treatment of hepatocellular carcinoma: results of the PRECISION V study. Cardiovasc. Intervent. Radiol. 33(1), 41-52 (2010).

54 Kalva SP, Pectasides M, Liu R et al. Safety and effectiveness of chemoembolization with drug-eluting beads for advanced-stage hepatocellular carcinoma. Cardiovasc. Intervent. Radiol. 37(2), 381-387 (2013).

55 Prajapati HJ, Xing M, Spivey JR et al. Survival, efficacy, and safety of small versus large doxorubicin drug-eluting beads TACE chemoembolization in patients with unresectable HCC. AJR. Am. J. Roentgenol. 203(6), W706-W714 (2014).

56 Lencioni R. Management of hepatocellular carcinoma with transarterial chemoembolization in the era of systemic targeted therapy. Crit. Rev. Oncol. Hematol. 83(2), 216-224 (2012).

57 Lencioni R, Petruzzi P, Crocetti L. Chemoembolization of hepatocellular carcinoma. Semin. Intervent. Radiol. 30(1), 3-11 (2013).

58 Zhu AX, Duda DG, Sahani D V, Jain RK. HCC and angiogenesis: possible targets and future directions. Nat. Rev. Clin. Oncol. 8(5), 292-301 (2011).

59 Wu XZ, Xie GR, Chen D. Hypoxia and hepatocellular carcinoma: the therapeutic target for hepatocellular carcinoma. J. Gastroenterol. Hepatol. 22(8), 1178-1182 (2007).

60 Minata M, Harada KH, Kudo M, Ikai I, Nishida N. The prognostic value of vascular endothelial growth factor in hepatocellular carcinoma for predicting metastasis after 
curative resection. Oncology 84(Suppl. 1), 75-81 (2013).

61 Wiggermann P, Wohlgemuth WA, Heibl M et al. Dynamic evaluation and quantification of microvascularization during degradable starch microspheres transarterial chemoembolisation (DSM-TACE) of HCC lesions using contrast enhanced ultrasound (CEUS): a feasibility study. Clin. Hemorheol. Microcirc. 53(4), 337-348 (2013).

62 Potente M, Gerhardt H, Carmeliet P. Basic and therapeutic aspects of angiogenesis. Cell 146(6), 873-887 (2011).

63 Forsberg JO. Transient blood flow reduction induced by intra-arterial injection of degradable starch microspheres: experiments on rats. Acta Chir. Scand. 144(5), 275-281 (1978).

64 Taguchi T. Chemo-occlusion for the treatment of liver cancer. A new technique using degradable starch microspheres. Clin. Pharmacokinet. 26(4), 275-291 (1994).

65 Nishiofuku H, Tanaka T, Matsuoka M et al. Transcatheter arterial chemoembolization using cisplatin powder mixed with degradable starch microspheres for colorectal liver metastases after FOLFOX failure: results of a Phase I/II study. J. Vasc. Interv. Radiol. 24(1), 56-65 (2013).

66 Furuse J, Ishii H, Satake M et al. Pilot study of transcatheter arterial chemoembolization with degradable starch microspheres in patients with hepatocellular carcinoma. Am. J. Clin. Oncol. 26(2), 159-164 (2003).

67 Kirchhoff TD, Rudolph KL, Layer G et al. Chemoocclusion vs chemoperfusion for treatment of advanced hepatocellular carcinoma: a randomised trial. Eur. J. Surg. Oncol. 32(2), 201-207 (2006).

68 Kirchhoff TD, Bleck JS, Dettmer A et al. Transarterial chemoembolization using degradable starch microspheres and iodized oil in the treatment of advanced hepatocellular carcinoma: evaluation of tumor response, toxicity, and survival. Hepatobiliary Pancreat. Dis. Int. 6(3), 259-266 (2007).

69 Yamasaki T, Hamabe S, Saeki I et al. A novel transcatheter arterial infusion chemotherapy using iodized oil and degradable starch microspheres for hepatocellular carcinoma: a prospective randomized trial.

J. Gastroenterol. 46(3), 359-366 (2011).

70 Niessen C, Unterpaintner E, Goessmann H et al. Degradable starch microspheres versus ethiodol and doxorubicin in transarterial chemoembolization of hepatocellular carcinoma. J. Vasc. Interv. Radiol. 25(2), 240-247 (2014).

71 Ingold JA, Reed GB, Kaplan HS, Bagshaw MA. Radiation hepatitis. Am. J. Roentgenol. Radium Ther. Nucl. Med. 93, 200-208 (1965).

72 Dawson LA, McGinn CJ, Normolle D et al. Escalated focal liver radiation and concurrent hepatic artery fluorodeoxyuridine for unresectable intrahepatic malignancies. J. Clin. Oncol. 18(11), 2210-2218 (2000).

73 Salem R, Lewandowski RJ, Atassi B et al. Treatment of unresectable hepatocellular carcinoma with use of $90 \mathrm{Y}$ microspheres (TheraSphere): safety, tumor response, and survival. J. Vasc. Interv. Radiol. 16(12), 1627-1639 (2005).

74 Salem R, Lewandowski RJ, Kulik L et al. Radioembolization results in longer time-to-progression and reduced toxicity compared with chemoembolization in patients with hepatocellular carcinoma. Gastroenterology 140(2), 497-507.e2 (2011).

75 Hilgard P, Hamami M, Fouly A El et al. Radioembolization with yttrium-90 glass microspheres in hepatocellular carcinoma: European experience on safety and long-term survival. Hepatology 52(5), 1741-1749 (2010).

76 Mazzaferro V, Sposito C, Bhoori S et al. Yttrium-90 radioembolization for intermediate-advanced hepatocellular carcinoma: a Phase 2 study. Hepatology 57(5), 1826-1837 (2013).

77 Kennedy AS, Nutting C, Coldwell D, Gaiser J, Drachenberg C. Pathologic response and microdosimetry of $(90) \mathrm{Y}$ microspheres in man: review of four explanted whole livers. Int. J. Radiat. Oncol. Biol. Phys. 60 (5), 1552-1563 (2004).

78 Kennedy A, Nag S, Salem R et al. Recommendations for radioembolization of hepatic malignancies using yttrium-90 microsphere brachytherapy: a consensus panel report from the radioembolization brachytherapy oncology consortium. Int
J. Radiat. Oncol. Biol. Phys. 68(1), 13-23 (2007).

79 Sangro B, Bilbao JI, Boan J et al. Radioembolization using 90Y-resin microspheres for patients with advanced hepatocellular carcinoma. Int. J. Radiat. Oncol. Biol. Phys. 66(3), 792-800 (2006).

80 Jung J, Yoon SM, Kim SY et al. Radiationinduced liver disease after stereotactic body radiotherapy for small hepatocellular carcinoma: clinical and dose-volumetric parameters. Radiat. Oncol. 8, 249 (2013).

81 Gil-Alzugaray B, Chopitea A, Iñarrairaegui M et al. Prognostic factors and prevention of radioembolization-induced liver disease. Hepatology 57(3), 1078-1087 (2013).

82 El Fouly A, Ertle J, El Dorry A et al. In intermediate stage hepatocellular carcinoma: radioembolization with yttrium 90 or chemoembolization? Liver Int. 35(2), 627-635 (2015).

83 Kim DY, Park BJ, Kim YH et al. Radioembolization with yttrium-90 resin microspheres in hepatocellular carcinoma: a multicenter prospective study. Am. J. Clin. Oncol. 38(5), 495-501 (2015).

84 Balkwill F, Mantovani A. Inflammation and cancer: back to Virchow? Lancet 357(9255), 539-545 (2001).

85 Coussens LM, Werb Z. Inflammation and cancer. Nature 420(6917), 860-867 (2002).

86 Nakahara Y, Mochiduki Y, Miyamoto Y, Nakahara Y, Katsura Y. Prognostic significance of the lymphocyte-to-neutrophil ratio in percutaneous fine-needle aspiration biopsy specimens of advanced nonsmall cell lung carcinoma. Cancer 104(6), 1271-1280 (2005).

87 Shimada H, Takiguchi N, Kainuma O et al. High preoperative neutrophil-lymphocyte ratio predicts poor survival in patients with gastric cancer. Gastric Cancer 13(3), 170-176 (2010).

88 Sukato DC, Tohme S, Chalhoub D et al. The prognostic role of neutrophil-to-lymphocyte ratio in patients with unresectable hepatocellular carcinoma treated with radioembolization. J. Vasc. Interv. Radiol. 26(6), 816-824.e1 (2015). 